Please do not remove this page

RMIT

UNIVERSITY

\title{
Adaptive algorithm for ternary filtering
}

Sadik, Amin; Hussain, Zahir; O'Shea, Peter

https://researchrepository.rmit.edu.au/esploro/outputs/9921862660701341/filesAndLinks?institution=61RMIT_INST\&index=null

Sadik, A., Hussain, Z., \& O'Shea, P. (2006). Adaptive algorithm for ternary filtering. Electronics Letters, 42(7), 420-421. https://doi.org/10.1049/el:20064257

Published Version: https://doi.org/10.1049/el:20064257

Repository homepage: https://researchrepository.rmit.edu.au

(c) IEE 2006. Personal use of this material is permitted. However, permission to reprint/republish this material for advertising or promotional purposes or for creating new collective works for resale or redistribution to servers or lists, or to reuse any copyrighted component of this work in other works must be obtained from the IEEE.

Downloaded On 2023/04/26 21:39:11 +1000 


\section{Adaptive algorithm for ternary filtering}

A.Z. Sadik, Z.M. Hussain and P. O'Shea

Despite their major advantage of hardware simplicity, ternary filters have limited usability in practice owing to their unresolved problem of adaptivity. This challenging problem is tackled by introducing an adaptive ternary LMS-like algorithm. Performance assessment using a sinusoidal input distorted by additive white Gaussian noise showed that the proposed algorithm is comparable to the traditional multi-bit Wiener LMS algorithm. This approach is expected to open the door for ternary systems to be ready for replacing multi-bit signal processing systems.

Introduction: Several recent works have made the theory of ternary filtering nearly mature and ready for application [1-5]. However, for a new filtering theory to be of large-scale application as a substitute for the traditional multi-bit DSP systems, efficient adaptive structures are inevitable. This is so since most applications are challenged by noise, distortion, and time-varying conditions. In fact, one of the major drawbacks that hindered analogue signal processing (ASP) for decades was lack of adaptivity.

Unfortunately, there is no adaptive LMS structure of any kind for ternary filtering. The challenge in this problem is the harsh quantisation that prevents straightforward LMS application. In this Letter we attempt to solve this problem by proposing an efficient structure for adaptive noise reduction. This application is of major significance in many applications, such as communication channel equalisation. The results were quite astonishing as the performance of this simple structure is comparable to that of the multi-bit LMS Wiener algorithm.

Adaptive ternary algorithm: We propose a structure inspired from the well-known LMS adaptive techniques. Fig. 1 illustrates the ternary structure that carries out the proposed adaptive algorithm. We assume that the received (observed) signal, $r(n)$, is in single-bit format that represents the digitised original signal $x(t)$ distorted by white Gaussian noise $\eta(t) \in \mathcal{N}\left(\sigma^{2}, 0\right)$. The same scenario in Fig. 1 can be used to represent a baseband version of a digital single-bit communication system with bandpass modulation [6].

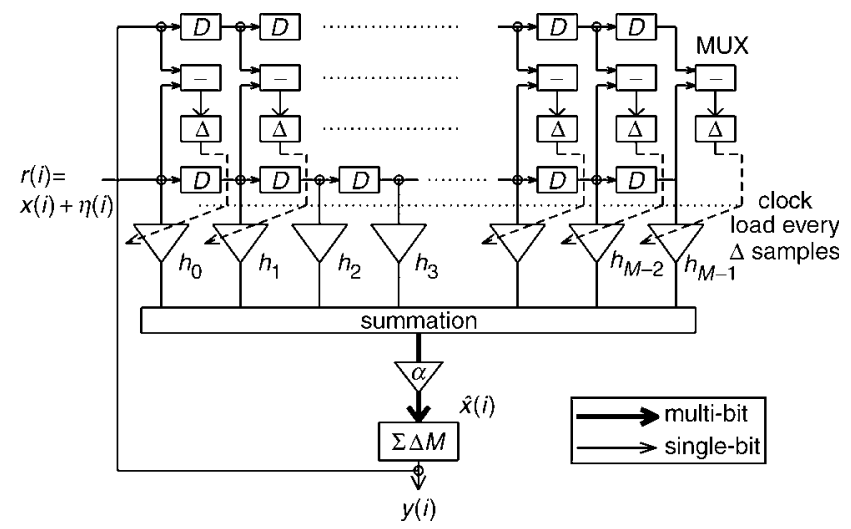

Fig. 1 Structure of adaptive ternary filter (note, ' $D$ ' represents single-bit delay element)

The symbol $\hat{x}(i)$ stands for the multi-bit estimated signal, and $y(i)$ is the estimated signal in single-bit format. The operation of this adaptive structure can be described as follows. The ternary system comprises $M$ adaptable taps and operates at an oversampling rate $R(R=64$, $128, \ldots$ ). This requirement has already been met as the input signal is assumed to be $\Sigma \Delta$ modulated. The single-bit estimated signal $y(i)$ is loaded sequentially into a shift register of length $M$, where the register content can be expressed by the vector:

$$
\mathbf{y}(i)=[y(i), \ldots, y(i-M-1)]
$$

Likewise, the regressor vector of the received single-bit signal will be: $\mathbf{r}(i)=[r(i), \ldots, r(i-M-1)]$, which is assumed to be in the form $\mathbf{r}=x+\eta$, where $x$ is the original signal vector in single-bit, and $\eta$ is the single-bit noise vector. This structure updates the ternary coefficients (taps) $\{h(j) \mid j=0,1,2, \ldots, M-1\}$ once every $\Delta$ samples, where
$\Delta$ is dependent on the oversampling ratio $R$, i.e. at Nyquist rate. This weights updating can be expressed as follows:

$$
\mathbf{h}_{n}=\frac{1}{2}\left(\mathbf{r}_{n}-\mathbf{y}_{n}\right)
$$

where $n=i \bmod \Delta$, and subscripts are used for time indexing instead of the brackets. The multi-bit estimated signal $\hat{x}$ at any instant $i$ is given by:

$$
\hat{x}_{i}=\alpha \mathbf{h}_{n-1}^{T} \mathbf{r}_{i}
$$

where $\alpha$ is a small positive parameter.

As $\hat{x}$ is in multi-bit format, a second-order standard $\Sigma \Delta$ modulator is used to convert $\hat{x}$ to a bit-stream (re-modulation). This $\Sigma \Delta$ stage should have a flat frequency response in the band of interest such that the information in $\hat{x}$ is maintained. However, this stage will inevitably introduce noise to the output $y_{i}$ owing to the quantisation error $Q_{i}$. In addition, to preserve stability of the system, the value of $\hat{x}$ should be maintained within the dynamic range of the $\Sigma \Delta$ modulator. This can be guaranteed by introducing the gain parameter $\alpha$, which is a small positive number. The parameter $\alpha$ is dependent on the oversampling ratio $(R)$ and the filter order at Nyquist rate $N$. This is so because the number of taps is proportional to $R$. For simplicity of implementation, $\alpha$ takes on negative powers of 2 .

The instantaneous single-bit estimated output $y_{i}$ is given as follows:

$$
y_{i}=\operatorname{sgn}\left(u_{i}\right)
$$

where $u_{i}$ is the quantiser input of the $\Sigma \Delta$ given by:

$$
u_{i}=2 u_{i-1}-u_{i-2}-2 y_{i-1}+y_{i-2}+\hat{x}_{i-1}
$$

From (1), the filter coefficients vector can be given as:

$$
\mathbf{h}_{n}=\frac{1}{2}\left[\mathbf{r}_{n}-\operatorname{sgn}\left(\mathbf{u}_{n}\right)\right]
$$

From (5), it is evident that elements of $\mathbf{h}_{n} \in\{0,+1,-1\}$.

Simulation and discussion: To assess the performance of the proposed adaptive ternary structure in terms of improvement in SNR, we attempt to compare it with that of a traditional LMS adaptive algorithm under similar circumstances. Fig. 2 shows the improvement in terms of the ratio $\rho=\mathrm{SNR}_{\mathrm{o}} / \mathrm{SNR}_{\mathrm{i}}$ against the $\mathrm{SNR}_{\mathrm{i}}$, where $\mathrm{SNR}_{\mathrm{o}}$ and $\mathrm{SNR}_{\mathrm{i}}$ denote the signal-to-noise ratio $(\mathrm{SNR})$ at the output and at the input of the system, respectively. The oversampling ratio is chosen as $R=128$, and the number of ternary coefficients is $M=2560$. The observed signal (input) $r_{i}$ is assumed to be the single-bit digitised version of the original sinusoid $x(t)$ which is distorted by additive white Gaussian noise $\eta(t)$. The sinusoid has an amplitude $A=0.5$ and a frequency $f_{o}=2000 \mathrm{~Hz}$.

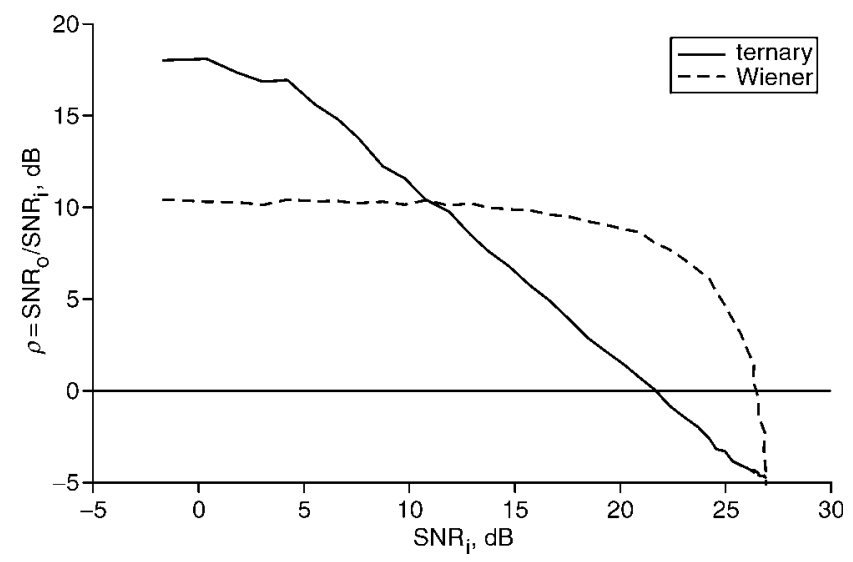

Fig. 2 SNR improvement using adaptive filtering: ternary against LMS using noisy sinusoid

We assume an adaptive LMS FIR filter with $N=R / M=20$ coefficients, operating on the same input signal. To be on the safe side, we assume the filter sampling rate as $4 \times$ Nyquist rate with infinite bit resolution. Moreover, the optimum $\mu$ (that gives minimum MSE) is used in this comparison. 
It is obvious from Fig. 2 that the adaptive ternary filter shows superior response (better $\rho$ ) when the input $\mathrm{SNR}, \mathrm{SNR}_{\mathrm{i}}$, is less than $12 \mathrm{~dB}$. On the other hand, the performance of the adaptive ternary algorithm deteriorates for $\mathrm{SNR}_{\mathrm{i}}>22 \mathrm{~dB}$ compared to the multi-bit LMS algorithm which exhibits better $\rho$ until $\mathrm{SNR}_{\mathrm{i}}=28 \mathrm{~dB}$.

This adaptive ternary structure is very efficient from a hardware implementation point of view, as the ternary taps can be realised by using simple multiplexers. Moreover, the updating rate $\Delta$ can be achieved through use of a conventional counter.

Discussion: As for LMS, filter coefficients are updated based on a weighted difference between the filter output (which is an estimation of the original signal) and the reference signal (chosen here to be a delayed version of the input signal itself). This is based on the assumption that noise and signal are uncorrelated; this is true for sinusoids and all narrowband signals. The estimation error (against iterations) converges in the LMS sense as in Fig. 3, similar to the conventional LMS. The system is stable as long as SDM is stable.

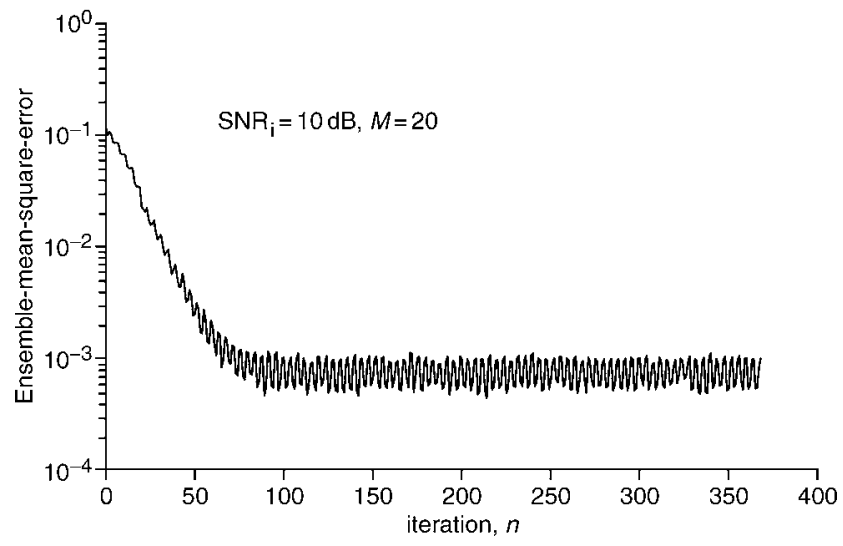

Fig. 3 Learning curve of proposed structure for noisy sinusoid
Conclusion: We have introduced an approach for adaptive ternary filtering. Despite the simple structure, simulation results showed that the proposed algorithm is parallel in performance to the standard multi-bit LMS algorithm. We expect this approach will open the door for a wide range of applications for ternary systems.

Acknowledgment: This work is supported by the Australian Research Council under ARC Discovery Grant DP0557429.

(C) IEE 2006

20 December 2005

Electronics Letters online no: 20064257

doi: 10.1049/el:20064257

A.Z. Sadik and Z.M. Hussain (School of Electrical \& Computer Engineering, RMIT University, Melbourne, Australia)

E-mail: zahir.hussain@rmit.edu.au

P. O'Shea (School of Engineering Systems, Queensland University of Technology, Brisbane, Australia)

\section{References}

1 Benvenuto, N., Franks, L.E., and Hill, F.S., Jr.: 'Realization of finite impulse response filters using coefficients $+1,0,-1$ ', IEEE Trans. Commun., 1985, 33, (10), pp. 1117-1125

2 Dick, C., and Harris, F.: 'FPGA signal processing using sigma-delta modulation', IEEE Signal Process. Mag., 2000, 17, pp. 20-35

3 Thompson, A.C., O'Shea, P., Hussain, Z.M., and Steele, B.R.: 'Efficient single-bit ternary digital filtering using sigma-delta modulator', IEEE Signal Process. Lett., 2004, 11, (2), pp. 162-166

4 Thompson, A.C., Hussain, Z.M., and O'Shea, P.: 'Efficient digital singlebit resonator', Electron. Lett., 2004, 40, (22), pp. 1396-1397

5 Sadik, A.Z., Hussain, Z.M., and O'Shea, P.: 'A single-bit digital DC-blocker using ternary filtering'. Proc. of IEEE TENCON 2005, Melbourne, Australia, November 2005

6 Proakis, J.G., et al.: 'Contemporary communication systems using Matlab’ (Brooks Cole, Pacific Grove, 2003, 2nd edn.) 